\title{
PROPRIEDADES PSICOMÉTRICAS DA ESCALA DE SATISFAÇÃO COM A VIDA NO CONTEXTO ESPORTIVO BRASILEIRO
}

\author{
PSYCHOMETRIC PROPERTIES OF SATISFACTION WITH LIFE SCALE IN THE BRAZILIAN SPORTS CONTEXT
}

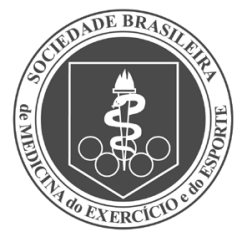

Artigo Original

Original ARTICLE Artículo Original

\section{PROPIEDADES PSICOMÉTRICAS DE LAESCALA DE SATISFACCIÓN CON LA VIDA ENEL CONTEXTO DEPORTIVO BRASILEÑO}

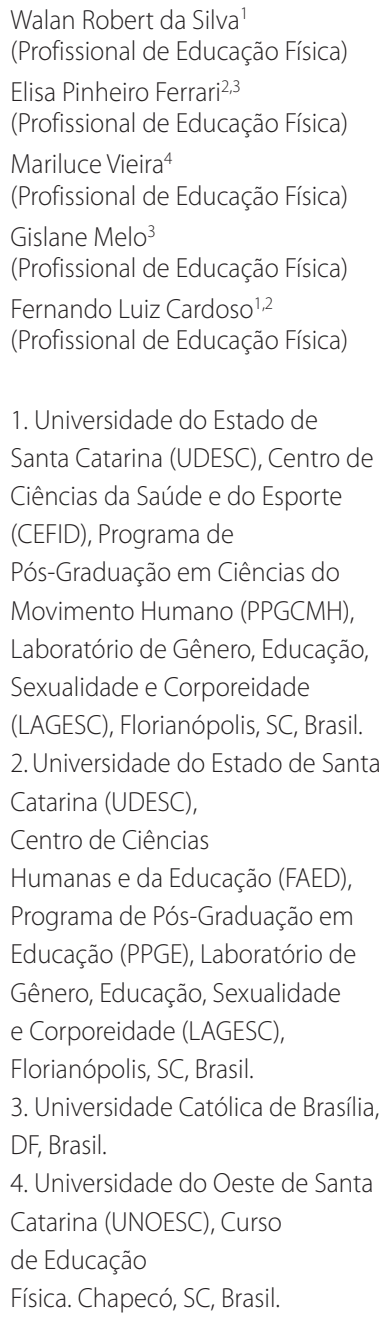

\section{Correspondência:}

Walan Robert da Silva.

Rua Pascoal Simone, 358, Coqueiros, Florianópolis, SC, Brasil, 88080-350 walanrobert@hotmail.com

\section{RESUMO}

Introdução: O bem-estar subjetivo é um construto referente à percepção que as pessoas têm de sua vida e à avaliação que dela fazem. Objetivo: O objetivo do estudo foi avaliar as propriedades psicométricas da Escala de Satisfação com a Vida no contexto esportivo brasileiro. Métodos: Participaram do estudo 593 atletas (371 homens), sendo 180 (30,4\%) praticantes de modalidades individuais e 413 (69,4\%) de modalidades coletivas. Por meio de análise fatorial exploratória e confirmatória, foi possível verificar que os itens carregaram em apenas um domínio, com índices de ajuste adequados (comparative fit index [CFI] =0,99; Tucker-Lewis Index [TLI] =0,98; standardized root mean square residual [SRMR] $=0,016$; standardized root mean square error of approximation $[$ RMSEA] $=0,026)$, confirmando o pressuposto de unidimensionalidade do construto. Resultados: Os resultados encontrados indicaram boa consistência interna $(a=0,804)$ e correlação estatisticamente significante entre todos os itens $(p<0,05)$. A análise de covariância demonstrou que a satisfação com a vida foi influenciada pela idade $(F[4,568] p<0,033)$, ou seja, a pontuação do instrumento variou em função da idade do respondente. Conclusão: Concluiu-se que a Escala de Satisfação com a Vida apresenta excelentes propriedades psicométricas para o uso em atletas, constituindo uma opção viável para a mensuração da satisfação com a vida no contexto esportivo. Nível de Evidência l; Teste de critérios diagnósticos desenvolvidos anteriormente em pacientes consecutivos (com padrão de referência "ouro" aplicado).

Descritores: Satisfação pessoal; Psicometria; Esportes.

\section{ABSTRACT}

Introduction: The subjective well-being is a construct referring to the perception that people have of their life and the evaluation that they make of it. Objective: The objective of this study was to evaluate the psychometric properties of the Life Satisfaction Scale in the Brazilian sport context. Methods: A total of 593 athletes (371 men) participated in the study, of which 180 (30.4\%) practiced individual modalities and 413 (69.4\%) collective modalities. By means of exploratory and confirmatory factor analysis, it was possible to verify that the items loaded in only one domain, with adequate adjustment indexes (comparative fit index [CFI] $=0.99$; Tucker-Lewis Index [TLI] $=0.98$; standardized root mean square residual [SRMR] $=0.016$; standardized root mean square error of approximation [RMSEA] $=0.026$ ), confirming the unidimensionality assumption of the construct. Results: The results showed good internal consistency $(a=0.804)$ and a statistically significant correlation between all items $(p<0.05)$. The covariance analysis showed that life satisfaction was influenced by age ( $F[4.568] p<0.033)$, that is, the instrument score varied according to the respondent's age. Conclusion: It was concluded that the Life Satisfaction Scale presents excellent psychometric properties for use in athletes, constituting a viable option for the measurement of satisfaction with life in the sport context. Level of Evidence l; Testing of previously developed diagnostic criteria on consecutive patients (with universally applied reference "gold" standard).

Keywords: Personal satisfaction; Psychometrics; Sports.

\section{RESUMEN}

Introducción El bienestar subjetivo es un constructo referente a la percepción que las personas tienen de su vida y la evaluación que de ella hacen. Objetivo: El objetivo del estudio fue evaluar las propiedades psicométricas de la Escala de Satisfacción de Vida en el contexto deportivo brasileño. Métodos: Participaron del estudio 593 atletas (371 hombres), siendo $180(30,4 \%)$ practicantes de modalidades individuales y $413(69,4 \%)$ de modalidades colectivas. Por medio de análisis factorial exploratorio y confirmatorio, fue posible verificar que los items cargaron en un solo dominio, con índices de ajuste adecuados, (comparative fit index [CFI] =0,99; Tucker-Lewis Index [TLI] =0,98; standardized root mean square residual [SRMR] $=0,016$; standardized root mean square error of approximation $[R M S E A]=0,026)$, confirmando el presupuesto de unidimensionalidad del constructo. Resultados: Los resultados encontrados indicaron buena consistencia interna $(\alpha=0,804)$ y correlación estadísticamente significativa entre todos los items $(p<0,05)$. El análisis de covariancia demostró que la satisfacción con la vida fue influenciada por la edad 
( $[$ [4,568] $p<0,033)$, es decir, la puntuación del instrumento varía en función de la edad del respondedor. Conclusión: Se concluyó que la Escala de Satisfacción con la Vida presenta excelentes propiedades psicométricas para el uso en atletas, constituyendo una opción viable para la medición de la satisfacción con la vida en el contexto deportivo. Nivel de Evidencia l; Pruebas de criterios de diagnósticos desarrollados anteriormente en pacientes consecutivos (con estándar de referencia "oro" aplicado).

Descriptores: Satisfacción personal; Psicometría; Deportes.

\section{INTRODUÇÃO}

O bem estar subjetivo trata-se de um construto referente à percepção que as pessoas têm acerca da sua vida e a avaliação que fazem da mesma. ${ }^{1}$ De forma geral, este é constituído por dois componentes, um afetivo referente aos aspectos emocionais e um cognitivo alusivo aos aspectos intelectuais, acerca da Satisfação com a Vida (SV). ${ }^{2-4}$

Partindo deste pressuposto, pode-se considerar a SV como um processo de juízo e avaliação geral da vida a partir da atribuição de valor a alguns domínios como, moradia, saúde, trabalho, entre outros. ${ }^{1,5}$ No entanto, em se tratando de um julgamento é necessário compreender que o mesmo resulta de uma comparação entre as circunstâncias de vida do sujeito e um padrão por ele estabelecido, uma referência, ${ }_{1}^{1}$ ou seja, para realizar um julgamento da sua própria vida, o mesmo é remetido a uma comparação com o meio social, cultural e histórico no qual está inserido, promovendo desta forma um maior interesse por parte dos estudiosos acerca do entendimento das razões que expliquem a variabilidade do fenômeno em diferentes culturas e contextos sociais, como o esporte.

Nesse sentido, estudos indicam que a SV de atletas está relacionada com múltiplos fatores como, traços afetivos, ${ }^{8}$ motivação para a prática esportiva, ${ }^{9}$ nível competitivo ${ }^{10}$ e planejamento de aposentadoria. ${ }^{11}$ Sendo assim, a SV é um tema crucial, pois pode ser um componente fundamental na obtenção de resultados esportivos positivos. ${ }^{12}$ Porém, apesar de sua importância, poucos estudos até a data atual investigaram a SV de atletas. ${ }^{8-12}$

Para estimular o desenvolvimento de pesquisas acerca da SV o desenvolvimento de instrumentos que avaliem a mesma se faz necessário, mediante um conhecimento profundo acerca do constructo em questão e os seus componentes. Desta forma, Glatzer ${ }^{13}$ sugeriu de acordo com a especificidade ou generalidade vivida, uma subdivisão para a SV em três níveis: (a) nível de satisfação diante de características específicas das condições particulares de vida; (b) nível de satisfação com a totalidade de conteúdos particulares da vida e (c) nível de satisfação com a totalidade dos domínios gerais de vida, denominada, exclusivamente, satisfação de vida ou satisfação com a vida em geral ou, ainda, satisfação de vida como um todo. Diante disso, surgiram diversos instrumentos, cujo objetivo se pauta na avaliação de uma das especificações mencionadas acima. Dentre os quais se destaca a Satisfaction With Life Scale (SWLS).

A SWLS está pautada no componente subjetivo do bem estar subjetivo e apresenta itens de natureza global distribuídos em uma única dimensão, cujo propósito se configura em avaliar a forma como as pessoas se julgam acerca do quanto estão satisfeitas com suas próprias vidas, tendo em conta seus interesses particulares e valores. ${ }^{14}$ É amplamente utilizada tanto no cenário científico internacional, ${ }^{8-12}$ quanto nacional, a qual foi adaptada e validada para diversas populações e países. ${ }^{15}$ No Brasil a SWLS foi traduzida e adaptada para adultos ${ }^{16}$ idosos $_{1}{ }^{17}$ estudantes ${ }^{18}$ e crianças. $^{19}$

Considerando os dados da literatura sobre a relação da SV em atletas com fatores que influenciam no esporte, não foi evidenciada a existência de um instrumento desenvolvido e validado para a avaliação da SV no Brasil. Assim, decidiu-se avaliar a SV de atletas brasileiros em diferentes modalidades coletivas e individuais, como também, testar algumas qualidades psicométricas da SWLS utilizada internacionalmente em diferentes grupos populacionais. Sendo assim, o objetivo do presente estudo foi avaliar as propriedades psicométricas da SWLS para o contexto esportivo brasileiro em termos de validade de construto, validade discriminante e de confiabilidade.

\section{MÉTODOS}

Trata-se de um estudo transversal não probabilístico, o qual faz parte de um projeto maior intitulado "Identidade Esportiva e Artística de Atletas e Bailarinos", o qual foi desenvolvido pelo Laboratório de Gênero, Educação, Sexualidade e Corporeidade (LAGESC) da Universidade do Estado de Santa Catarina (UDESC). Submetido e aprovado pelo Comitê de Ética em Pesquisas com Seres Humanos (CEPSH) da UDESC sob o número de protocolo: 275.381/2013.

Participaram do estudo 593 atletas de ambos os sexos, 62.6\% ( $n=371)$ homens e 37.4\% ( $n=222)$ mulheres, com média de idade de $21.2( \pm 5.6)$ anos. Destes, 30.4\% ( $n=180)$ praticavam modalidades individuais, atletismo ( $n=15)$, ginástica artística $(n=3)$, natação $(n=7)$, judô $(n=63)$, karatê $(n=2)$, taekwondo $(n=6)$, jiu-jitsu $(n=31)$, ciclismo $(n=4)$, tênis de mesa $(n=39)$, xadrez $(n=10)$; e 69.4\% ( $n=413)$ modalidades coletivas, futebol de campo ( $n=64)$, futsal $(n=138)$, da mesorregião Oeste de Santa Catarina (Brasil). Os participantes foram escolhidos de forma intencional a partir, dos seguintes critérios de inclusão: idade mínima de 16 anos, estar federado por um clube, associação ou secretaria de esporte por no mínimo um ano, treinar de forma sistematizada a pelo menos um ano em uma frequência mínima de três vezes por semana e estar treinando regularmente no período da coleta de dados. A coleta de dados foi realizada nos meses de agosto a novembro de 2014. Ressalta-se que todos os participantes da pesquisa preencheram o Termo de Consentimento Livre Esclarecido (TCLE). Na Tabela 1 são apresentadas todas as características esportivas coletadas sobre os atletas.

A SWLS foi proposta por Diener et al. ${ }^{14}$ com o objetivo de avaliar quão satisfeitas as pessoas estão com suas vidas. Este instrumento foi criado com base na teoria do bem estar subjetivo ${ }^{2}$, mais precisamente com o intuito de avaliar o componente cognitivo da satisfação com a vida, por meio de cinco itens, com opções de resposta variando de 1 (discordo totalmente) a 7 (concordo plenamente), sendo que, quanto mais próxima de 7 é a resposta do indivíduo mais elevados são os seus índices de SV. ${ }^{14}$

Tabela 1. Caracterização dos participantes do estudo, quanto aos aspectos esportivos.

\begin{tabular}{c|c}
\hline Variáveis & $\mathbf{X}(\mathbf{d p})$ \\
\hline Tempo de prática sistematizada & $8,17(3,1)$ \\
\hline Frequência semanal de treino & $4,7(1,1)$ \\
\hline Duração do treino em horas & $2,4(1,2)$ \\
\hline Nível competitivo & $\mathrm{n}(\%)$ \\
\hline Estadual & $476(80,3)$ \\
\hline Nacional & $77(13,0)$ \\
\hline Internacional & $40(6,7)$ \\
\hline
\end{tabular}


No presente estudo, foi utilizada a versão em português da SWLS, traduzida por Gouveia et al. ${ }^{16}$ por meio do processo de "back translation" da escala original. Além, do processo de tradução foram testadas a fidedignidade, a validade de conteúdo e de critério em brasileiros. A partir deste estudo pode-se verificar que, a versão brasileira do SWLS é um instrumento válido para o uso em adultos, sendo que os itens carregaram em um único fator confirmando a teoria e explicando $66 \%$ da variância total do construto e uma boa confiabilidade (Alfa de Cronbach=0.87). ${ }^{19}$

Foi incluído um questionário contendo informações gerais, referentes ao nome, idade, sexo, e modalidade esportiva praticada, que foi classificada em dois grandes grupos para fins estatísticos, "modalidades coletivas" cuja referência se pauta na interação entre os componentes/ atletas, sendo estas, "futebol de campo", "futsal", "voleibol", "handebol" e "basquetebol" e "modalidades individuais", aquelas com provas individuais, quando o sujeito participa sozinho durante a ação esportiva total, as quais foram: "atletismo", "ginástica artística", "natação", "judô", "karatê", "taekwondo", "jiu-jitsu", "ciclismo", "tênis de mesa" e "xadrez"28. Os questionários foram auto aplicados nas dependências dos locais de treinamento. Foi recomendado aos atletas que não conversassem durante o preenchimento dos questionários.

\section{Análise dos dados}

Inicialmente, a fim de caracterizar os participantes foi realizada uma estatística descritiva, por meio de frequência relativa e absoluta e valores de média e desvio padrão. A validade de construto foi avaliada mediante a realização da análise fatorial exploratória com rotação Varimax, considerando um índice de carga fatorial de 0.3 para exclusão de itens ${ }^{20} \mathrm{e}$ teste de KMO e esfericidade de Bartlett's para verificar a adequação da análise fatorial. A consistência interna foi avaliada utilizando o Alpha de Cronbach, correlação Policórica e a fidedignidade pelo Índice de Correlação Intraclasse (ICC). Ainda foi empregada a análise de covariância (ANCOVA) para identificar a capacidade do instrumento em discriminar os atletas quanto à idade, o sexo biológico e o tipo de modalidade esportiva praticada (modalidades individuais ou coletivas). Para todos os testes o nível de significância adotado foi de $5 \%$. Os dados foram tabulados e analisados no Statistical Package for the Social Sciences (SPSS) (versão 20.0, SPSS Inc., Chicago, Illinois, USA).

A análise fatorial confirmatória foi realizada através do programa estatístico STATA (versão 13.1 Akeway Drive, College Station, Tx, Canadá) - Para verificar a adequação do modelo foram considerados os seguintes parâmetros, Qui-quadrado $\left(x^{2}\right)$; Comparative Fit Index (CFI); Tucker Lewis Index (TLI); Root Mean Square Error of Approximation (RMSEA). Os valores de referência considerados para o Qui-quadrado $\left(x^{2}\right)$ foi um valor de $p$ não significativo, ${ }^{21} 0.95$ como um valor mínimo para inferir o ajuste do modelo, de acordo com os índices CFI e TLI, ${ }^{22}$ enquanto para o RMSEA valores de zero a 0.08 como indicativo de ajuste aceitável. ${ }^{22}$

\section{RESULTADOS}

O escore médio obtido na SWLS nos atletas avaliados foi de $24.2( \pm 5.1)$ indicando que os participantes do estudo têm uma boa percepção da SV (dados não apresentados).

Quanto à análise fatorial da escala, um valor de teste KMO de 0.833 e do Teste de Bartlett $(p<0.001)$ indicaram que os dados foram adequados para a extração de fatores. Tal análise revelou um único fator representando $57.6 \%$ da variância (Tabela 2).

O gráfico Scree Plots confirma mais uma vez que o instrumento analisado carrega em um único fator. (Figura 1)

exploratória da SWLS adaptada para o contexto esportivo.

Na Tabela 2 são apresentados os valores do Alfa de Cronbach para os cincos itens da escala e os valores de cada item individualmente.
Tabela 2. Análise Fatorial Exploratória da SWLS adaptada para o contexto esportivo com o Método dos Componentes Principais e Rotação Varimax.

\begin{tabular}{c|c}
\hline \multicolumn{1}{c|}{ Itens da escala } & Fator \\
\hline 1. Na maioria dos aspectos, minha vida é próxima ao meu ideal. & 0,809 \\
\hline 2. As condições da minha vida são excelentes. & 0,773 \\
\hline 3. Estou satisfeito com minha vida. & 0,809 \\
\hline 4. Dentro do possível, tenho as coisas importantes que & 0,751 \\
\hline $\begin{array}{c}\text { 5. Se puero na vida. } \\
\text { minha vida. }\end{array}$ & 0,640 \\
\hline
\end{tabular}

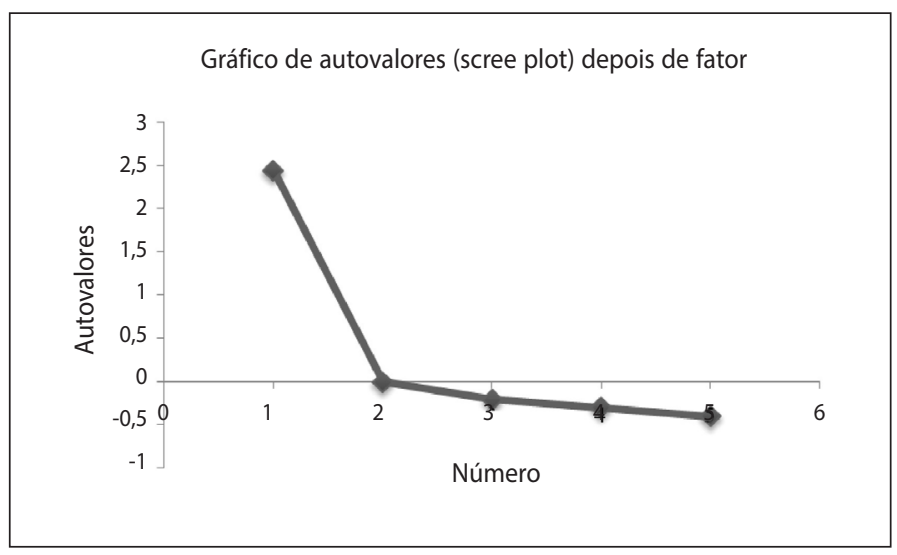

Figura 1. Gráfico Scree dos autovalores obtidos na análise fatorial exploratória da SWLS adaptada para o contexto esportivo.

A fim de verificar alterações no valor total de alfa, cada item foi retirado da escala alternativamente. Como não ocorreu alteração significativa, a análise fatorial exploratória não indicou a retirada de nenhum dos itens. (Tabela 3) A análise de fidedignidade da escala indicou um valor de ICC de 0.804 (dados não apresentados).

A consistência interna foi testada por meio da correlação Policórica entre os itens da escala. Segundo os dados apresentados na Tabela 3 todos os itens se correlacionaram significativamente $(p<0.05)$, confirmando uma boa consistência interna do instrumento. (Tabela 4)

Os valores da análise de covariância demonstraram que a pontuação da escala SWLS adaptada para o contexto esportivo varia em função da idade do sujeito ( $F[4.568]$; $p<0.05)$, mas não em função do tipo de modalidade praticada ( $F[0.487] ; p<0.05)$ coletivo $(24.4 \pm 5.5)$ individual

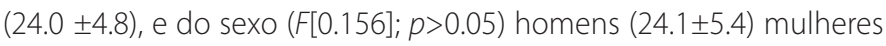
(24.3 \pm 4.6$)$. Não foi constatado efeito de interação significativo entre essas três variáveis.

Ao analisar a estrutura fatorial do Modelo constituído por um fator da versão brasileira do SWLS aplicado no contexto esportivo, verificou-se que o valor corrigido do $x^{2}$ não foi significativo $p>0.05$, o que sugere um forte ajuste. Bem como, valores adequados para os índices de ajustamento: TLI (0.98), CFI (0.99), índice da RMSEA (0.026) e o índice SRMR (0.016). (Tabela 5)

A Figura 2 representa a estrutura graficamente confirmada da SWLS adaptada para o contexto esportivo brasileiro.

\section{DISCUSSÃO}

Ao aplicar a SWLS para o contexto esportivo brasileiro, os participantes desse estudo apresentaram um escore médio de 24.2 ( \pm 5.1 ). Ao comparar este, com o obtido por outras pesquisas ${ }^{15}$ pode-se verificar que os atletas investigados apresentaram maior satisfação com a vida em relação a estudantes americanos, ${ }_{1}^{14}$ chineses, ${ }^{23}$ atletas franceses ${ }^{24}$ e valores similares ao observado em adultos americanos. ${ }^{3}$ De forma geral, a pontuação obtida pela SWLS pode ser interpretada sob a forma 
Tabela 3. Valor total e alteração do valor total do Alfa de Crombach com a exclusão alternada dos itens da SWLS adaptada para o contexto esportivo.

\begin{tabular}{c|c}
\hline Itens da escala & Alfa \\
\hline Valor total da escala & 0,804 \\
\hline Se o item 1 for excluído & 0,750 \\
\hline Se o item 2 for excluído & 0,760 \\
\hline Se o item 3 for excluído & 0,746 \\
\hline Se o item 4 for excluído & 0,764 \\
\hline Se o item 5 for excluído & 0,813 \\
\hline
\end{tabular}

Tabela 4. Correlação entre os itens do instrumento e o escore total da SWLS adaptada para o contexto esportivo.

\begin{tabular}{|c|c|c|c|c|c|c|}
\hline & $\begin{array}{c}\text { Item } 1 \\
(r)\end{array}$ & $\begin{array}{c}\text { Item } 2 \\
(r)\end{array}$ & $\begin{array}{c}\text { Item } 3 \\
(\mathrm{r})\end{array}$ & $\begin{array}{c}\text { Item4 } \\
(\mathrm{r})\end{array}$ & $\begin{array}{c}\text { Item } 5 \\
(\mathrm{r})\end{array}$ & $\begin{array}{l}\text { Escore } \\
\text { total(r) }\end{array}$ \\
\hline Item 1 & 1 & $0,561^{* *}$ & $0,601^{* *}$ & $0,506^{* *}$ & $0,348^{* *}$ & $0,778^{* *}$ \\
\hline Item 2 & $0,561^{* *}$ & 1 & $0,533^{* *}$ & $0,439^{* *}$ & $0,374^{* *}$ & $0,753^{* *}$ \\
\hline Item 3 & $0,601^{* *}$ & $0,533^{* *}$ & 1 & $0,492^{* *}$ & $0,398^{* *}$ & $0,789^{* *}$ \\
\hline Item 4 & $0,506^{* *}$ & $0,439^{* *}$ & $0,492^{* * *}$ & 1 & $0,415^{* *}$ & $0,749^{* *}$ \\
\hline Item 5 & $0,348^{* *}$ & $0,374^{* *}$ & $0,398^{* * *}$ & $0,415^{* *}$ & 1 & $0,711^{* *}$ \\
\hline Escore total & $0,778^{* *}$ & $0,753^{* *}$ & $0,789^{* *}$ & $0,749^{* *}$ & $0,711^{* *}$ & 1 \\
\hline
\end{tabular}

Tabela 5. Índices de Ajustamento SWLSadaptada para o contexto esportivo.

\begin{tabular}{c|c|c|c|c|c}
\hline $\mathbf{X}^{2}$ & $\mathbf{X}^{2}$ /df & CFI & TLI & RMSEA & SRMR \\
\hline 5,581 & 0,233 & 0,993 & 0,981 & 0,026 & 0,016 \\
\hline
\end{tabular}

X2/df: Qui-quadrado/graus de liberdade; CFI: Comparative Fit Index; RMSEA: Root Mean Square Error of Approximation; SRMR: Standardized Root Mean-Squared Residual; TLI/NNFI:Tucker-Lewis Index/Non-normed fit index; IFI: Bollen's Incremental Fit Index.

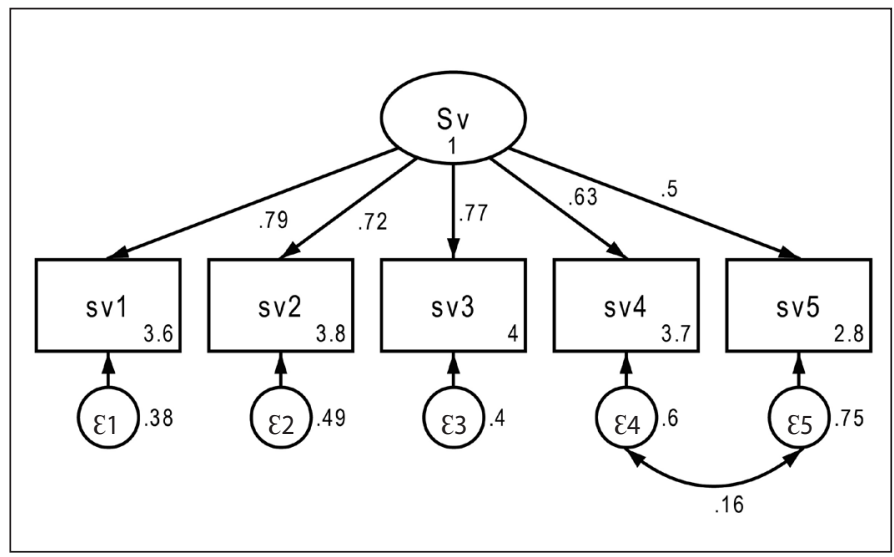

Figura 2. Analise fatorial confirmatória da SWLS adaptada para o contexto esportivo

de valores absolutos e categóricos, sendo que uma pontuação de 20 pontos representa o ponto neutro da escala, em que o entrevistado demonstra estar igualmente satisfeito e insatisfeito com a sua vida. O escore de satisfação com a vida encontrado no presente estudo vai ao encontro com o observado nos estudos de Baldin et al. ${ }^{24} \mathrm{com}$ atletas franceses, Wilson ${ }^{10}$ com atletas universitários americanos e Batista et al. ${ }^{9}$ com atletas portugueses veteranos, os quais observaram, escores de satisfação com a vida acima do ponto neutro em relação às demais populações.

O SWLS é um instrumento amplamente utilizado em diversos países $^{15}$. No entanto, a partir de uma revisão preliminar de literatura não foram encontradas validações desta para o contexto esportivo a nível nacional e internacional. Diante disso, os resultados encontrados pelo presente estudo vêm a contribuir com o avanço do conhecimento na área, disponibilizando um instrumento de satisfação com a vida, validado para o contexto esportivo.
A SWLS foi desenvolvida por Diener et al. ${ }^{14}$ para avaliar o nível de satisfação geral de uma pessoa com suas vidas. A partir da análise fatorial da versão brasileira da escala aplicada ao contexto esportivo, pode-se verificar que todos os itens apresentaram cargas de alto fator de forma unidimensional, corroborando com a escala original e com o resultado encontrado por outros estudos nacionais que validaram a escala em diversas populações como, idosos. ${ }^{17}$

Dentre todos os itens que compõem a escala investigada, o quinto "Se pudesse viver uma segunda vez, não mudaria quase nada em minha vida", foi o que apresentou a menor carga fatorial. Este resultado corrobora ao observado por Albuquerque et al. ${ }^{17}$ ao validar a escala para idosos brasileiros, os quais sugerem uma reestruturação desse item justificando uma má compreensão do mesmo por parte dos participantes da pesquisa e que pode ser atribuído a uma formação negativa da questão ("não mudaria quase nada na minha vida").

Quanto à consistência interna encontrada, a SWLS apresentou índices adequados para a sua aplicação no contexto esportivo (alpha= 0.804) e corrobora ao obtido por outros estudos. ${ }^{15}$ Blais et al..$^{25}$ obtiveram valores entre 0.79 a 0.84 ao aplicar o instrumento na população adulta canadense. Enquanto, Gouveia et al. ${ }^{16}$ verificaram um alfa de 0.89 em médicos brasileiros. Estes dados demonstram que independente da amostra investigada o instrumento em questão, a SWLS, apresenta uma boa consistência interna, podendo ser considerado um traço latente. Ressalta-se, que ao analisar o alpha de Cronbach com a exclusão do item cinco da escala, o que apresentou menor carga fatorial, a consistência interna do instrumento se manteve estável, justificando assim, a manutenção do item na escala.

Mediante a presença de correlações estatisticamente significantes entre os itens entre si e dos mesmos com o escore total da escala, pode-se dizer que todos os que compõem o instrumento avaliam um mesmo constructo, a satisfação com a vida, suportado por meio do teste da estrutura unifatorial da escala aplicada em atletas brasileiros. Sendo que, os indicadores de ajuste encontrados são favoráveis à identificação de um único fator, com todos os itens apresentando saturações estatisticamente diferentes de zero, comprovando, portanto que esta estrutura é inequívoca. ${ }^{15}$

Em relação à influência da idade na satisfação com a vida, os resultados do presente estudo indicaram que quanto mais velho o atleta maior sua satisfação com a vida corroborando com a maioria das investigações, as quais sugerem que com o passar da idade a satisfação com a vida tende a aumentar. ${ }^{15}$ Segundo Emerson et al. ${ }^{15}$ a satisfação com a vida é influenciada por variáveis como a maturidade e as realizações profissionais, à medida que ocorre um acréscimo nestas, a satisfação com a vida tende a aumentar também. Afinal, as pessoas mais velhas, em princípio, gozam de mais estabilidade financeira e realização pessoal, o que thes permite inclusive maior otimismo diante da vida.

A satisfação com a vida não foi influenciada pelo sexo dos atletas, o que vai ao encontro com o evidenciado em uma revisão sistemática recente. ${ }^{15}$ Isto é, no aspecto cognitivo do bem estar subjetivo, que diz respeito à satisfação com a vida, o comportamento entre homens e mulheres é similar. Todavia, esta é apenas uma hipótese necessitando que se considere em estudos futuros os modos de enfrentamento que os atletas de ambos os sexos adotam diante dos aspectos afetivos.

Ressalta-se a partir do presente estudo, a importância de se elaborar estudos que visem compreender o construto SV em diferentes estratos da sociedade, principalmente em diferentes amostras esportivas, a fim de identificar a influência desse construto no desempenho esportivo em diferentes modalidades e na vida dos atletas de diferentes faixas etárias. 


\section{CONCLUSÃO}

Em conclusão, de forma geral esse estudo comprova uma maior SV em atletas em relação aos demais grupos sociais, sendo que, a versão da escala de satisfação com a vida na composição de cinco itens mostrou um bom desempenho psicométrico quando estudado a partir de uma amostra de atletas. $\mathrm{O}$ instrumento em questão apresentou atributos satisfatórios de validade de conteúdo e consistência interna. Ressalta-se, que no contexto brasileiro não se identificou outro instrumento específico que avalie características da satisfação com a vida relacionada com o esporte. Desta forma, a disponibilidade deste, favorece o desenvolvimento e a efetividade das intervenções psicológicas em relação ao esporte como já acontece em outros países. Além disso, destaca-se que mesmo a escala sendo desenvolvida para outras populações e contextos, os resultados encontrados confirmam a validade para o emprego em atletas brasileiros. Porém, alguns aspectos regionais e socioculturais merecem ser analisados com maior sagacidade, a fim de que a escala possa ter a abrangência a que se propõe. Com isso sugerem-se a realização de outras investigações em âmbito nacional no intuito de sanar estas dúvidas.

Todos os autores declararam não haver qualquer potencial conflito de interesses referente a este artigo.

CONTRIBUIÇÕES DOS AUTORES: Cada autor contribuiu individual e significativamente para o desenvolvimento do manuscrito. WRS $(0000-0003-0568-4272)^{*}$ e MPV(00000002-9352-0878)*: foram os principais contribuintes na redação do manuscrito; EPF(0000-0001-9346-1460)*: avaliou os dados da análise estatística; GM (0000-0003-3551$5963)^{*}$ e FLC (0000-0002-3074-0988)*: realizaram a revisão do manuscrito e contribuíram com o conceito intelectual do estudo. *ORCID (Open Researcher and Contributor ID).

\section{REFERÊNCIAS}

1. Albuquerque AS, Tróccoli BT. Desenvolvimento de uma escala de bem-estar subjetivo. Psic Teo Pesq. 2004;20(2); 153-64

2. Diener E. Subjective well-being. Psychol Bull. 1984;95(3);542-95.

3. Diener E, Suh EM, Lucas RE, Smith HL. Subjective well-being: three decades of progress. Psychol Bull.1999;125(2):276-302.

4. Diener E, Biswas-Diener R. New directions in subjective well-being research: the cutting edge. Journal of Clinical Research. 2000;31:103-57.

5. Emmons RA. Personal strivings: an approach to personality and subjective well-being. J Pers and Soc Psychol. 1986;51(5):1058-68

6. Scorsolini-Comin F, Santos MA. Satisfação com a vida e satisfação diádica: correlações entre construtos de bem-estar. Psico-USF. 2011;15(2):249-56

7. Scorsolini-Comin F, Santos MA. A medida positiva dos afetos: bem-estar subjetivo em pessoas casadas. Psicol Reflex Crít. 2012:25(1):11-20

8. Chen LH, Kee YH. Gratitude and adolescent athletes' well-being. Soc Indic Res. 2008;89(2):361-73.

9. Batista MA, Castuera RJ, Roman,ML, Carrón,MI, Muñoz SL. Self-determined motivation and life satisfaction in portuguese veterans athletes. Retos. 2017;(32): 124-9.

10. Wilson M. Differences in depression, anxiety, and life satisfaction between intercollegiate athletes, intramural participants, and non-athletes Masters Theses \& Specialist Projects. The Faculty of the Department of Psychology Western Kentucky University Bowling Green. Kentucky:2016.

11. Stambulova N, Stephan Y, Jäphag U. Athletic retirement: a cross-national comparison of elite French and Swedish athletes. Psychol Sport Exerc. 2007;8(1):101-18.

12. Chen $\mathrm{LH}, \mathrm{Wu} \mathrm{CH}$, Lin $\mathrm{SH}, \mathrm{YeYC}$. Top-down or bottom-up? the reciprocal longitudinal relationship between athletes'Team Satisfaction and Life Satisfaction. Sport, Exercise, and Performance Psychology. 2017.
13. Glatzer W. Subjective well-being. Soc Indic Res. 1987;19:25-38.

14. Diener E. Emmons RA, Larsen RJ, Griffin S. The satisfaction with life scale. Journal Pers Assess. $1985 ; 49(1): 71-5$.

15. Emerson SD, Guhn M, Gadermann,A. Measurement invariance of the satisfaction with life scale: reviewing three decades of research. Quali. Life Res. 2017.

16. Oliveira GF, Barbosa GA, Souza LE, Costa CL, Araújo RC, Gouveia W. Satisfação com a vida entre profissionais da saúde: correlatos demográficos e laborais. Rev Bioét. 2009;17(2):319-34.

17. Albuquerque FB, Sousa FM, Martins CR. Validação das escalas de satisfação com a vida e afetos para idosos rurais. Psico. 2010;41(1);85-92.

18. Giacomoni CH. Bem-estar subjetivo: em busca da qualidade de vida. Temas em Psicol. 2004;12(1):43-50.

19. Giacomoni CH, Hutz CS. Escala multidimensional de satisfação da vida para crianças: estudos de construção e validação. Est Psicol. 2008;25(1):23-35.

20. Damásio BF. Uso da análise fatorial exploratória em psicologia. Aval Psicol. 2012;11(2):213-28.

21. Hu LT, Bentler PM. Cutoff criteria for fit indexes in covariance structure analysis: conventional criteria versus new alternatives: structural equation modeling:. Multidisciplinary J. 1999;6(1):1-55.

22. Browne MW, Cudeck R. Alternative ways of assessing model fit. Sage Focus Editions.1993;154:136

23. Li JB, Delvecchio E, Lis A, Nie YG, Di Riso D. Positive coping as mediator between self-control and life satisfaction: evidence from two Chinese samples. Pers Indiv Differ. 2016; 97:130-3.

24. Baudin N, Aluja A, Rolland JP, Blanch A. The role of personality in satisfaction with life and sport. Psicologia Conductual. 2011;19(2): 333-45

25. Blais MR, Vallerand RJ, Pelletier LG, Briere NM. L'echelle de satisfaction de vie: validation canadienne-francaise du «Satisfaction With Life Scale». Can J Beh Sci. 1989;21 (2):210-23. 